\title{
Dynamic flow alterations dictate leukocyte adhesion and response to endovascular interventions
}

\author{
Yoram Richter, ${ }^{1}$ Adam Groothuis, ${ }^{1}$ Philip Seifert, ${ }^{1}$ and Elazer R. Edelman ${ }^{1,2}$ \\ ${ }^{1}$ Harvard-M.I.T. Division of Health Sciences and Technology, Massachusetts Institute of Technology, Cambridge, Massachusetts, USA. \\ 2Department of Medicine, Cardiovascular Division, Brigham and Women's Hospital and Harvard Medical School, Boston, Massachusetts, USA.
}

\begin{abstract}
Although arterial bifurcations are frequent sites for obstructive atherosclerotic lesions, the optimal approach to these lesions remains unresolved. Benchtop models of arterial bifurcations were analyzed for flow disturbances known to correlate with vascular disease. These models possess an adaptable geometry capable of simulating the course of arterial disease and the effects of arterial interventions. Chronic in vivo studies evaluated the effect of flow disturbances on the pattern of neointimal hyperplasia. Acute in vivo studies helped propose a mechanism that bridges the early mechanical stimulus and the late tissue effect. Sidebranch (SB) dilation adversely affected flow patterns in the main branch (MB) and, as a result, the long-term $\mathrm{MB}$ patency of stents implanted in pig arteries. Critical to this effect is chronic MB remodeling that seems to compensate for an occluded SB. Acute leukocyte recruitment was directly influenced by the changes in flow patterns, suggesting a link between flow disturbance on the one hand and leukocyte recruitment and intimal hyperplasia on the other. It is often impossible to simultaneously maximize the total cross-sectional area of both branches and to minimize flow disturbance in the MB. The apparent trade-off between these two clinically desirable goals may explain many of the common failure modes of bifurcation stenting.
\end{abstract}

\section{Introduction}

The defining feature of the vascular tree is its repeated bifurcations. By virtue of their prevalence and inherently disturbed flow, these are frequent sites of atherosclerotic disease (1-4). Arterial bifurcations are nearly always asymmetric and can be divided into a main branch (MB) and a (usually smaller) side branch (SB). Atherosclerotic disease can manifest as luminal narrowing of the MB, the SB, or both (5). An important clinical question is the optimal course of treatment of a bifurcation (5-12). As the technology and technique of endovascular interventions advances, bifurcations are increasingly being confronted. While occluded MBs are dilated in nearly all cases, the approach to the SB is unclear. More specifically, it has not yet been determined what the implications of dual MB and SB dilation are as opposed to dilation of the $\mathrm{MB}$ alone. Clinical experience has shown that bifurcation interventions do relatively poorly with a substantially higher rate of restenosis (reocclusion of the vascular lumen within months after intervention) when compared with nonbifurcating geometries $(13,14)$. Paradoxically, while stenting of individual lesions has been shown to be superior to balloon angioplasty, stenting of both branches of a bifurcation offers no advantage over stenting of the $\mathrm{MB}$ alone (15). Part of the explanation for the perceived failure of bifurcation stenting is the increased technical challenge that a bifurcation intervention poses to the cardiologist. As the dedicated techniques and devices $(16,17)$ designed for these bifurcation interventions improve, technical difficulties will be alleviated. This raises the question whether there are mechanistic variables inherent to bifurcations that lead to poor clinical results irrespective of technique.

Nonstandard abbreviations used: main branch (MB); poly(ethylene glycol) (PEG); side branch (SB).

Conflict of interest: The authors have declared that no conflict of interest exists.

Citation for this article: J. Clin. Invest. 113:1607-1614 (2004).

doi:10.1172/JCI200421007.
Hemodynamics has long been identified as a major influence in the formation and localization of atherosclerotic plaques (1, 18-23). More recent works have focused primarily on regions of flow separation $(2,4)$. These are areas where the streamlines curve away from the vessel wall only to curve back toward the wall more distally, creating a recirculating eddy. Regions of boundary layer separation and the associated introduction of low (3) and oscillatory (4) shear stress are powerful atherogenic stimuli (24-27). This association has motivated the investigation of vascular flow patterns using in vitro flow models $(1,18-20,25,28-31)$. Although previous flow models are extensive, they all share an important limitation: Modeling of a static geometry is useful in identifying regions that are at an increased risk; however, it does not reflect the true dynamic nature of an arterial system that compensates for and minimizes any disturbance. Thus the very results obtained from a static model dictate that vascular remodeling is in place and will continue, reducing the relevancy of that very same model. We hypothesized that these dynamic processes play a critical role in vascular response to injury. Furthermore, we hypothesized that there are interactions between different sites in the arterial tree - in other words, that an intervention at one site will alter the flow pattern at another. Specifically, we propose that dilation of a bifurcation SB could have an adverse effect on MB flow pattern and in so doing impair long-term MB patency.

Over the course of progression of vascular disease in the SB of a bifurcation, the geometry of the MB dynamically compensates for the globally altered flow conditions (32). By the time the SB becomes occluded (which can take several decades), the MB will have been optimized to functioning in the SB-occluded rather than the SB-patent state. Thus, the virtually instantaneous dilation of the SB that occurs during angioplasty does not return the bifurcation to its original healthy state. Rather, a new state is formed whereby both branches are patent, but the geometry of the 


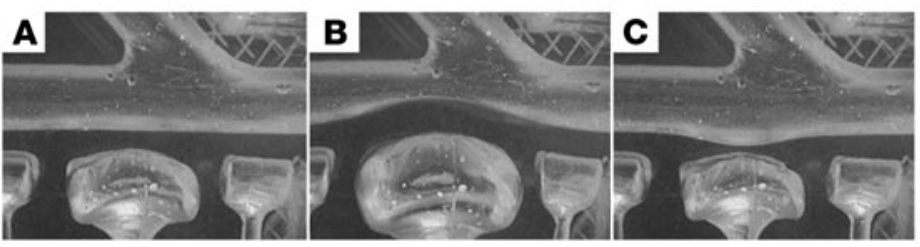

Figure 1

Dynamic control of model geometry. The silicone flow channel (right to left at top) is an idealized bifurcation. (A) In the native state, the lumens are left intact. The pneumatic drivers seen on the bottom of this photo, for example, can impinge on the lumen to simulate lesions of variable eccentricity $(\mathbf{B})$ to create taper or to locally expand by controlled suction (C). By combining several of these drivers of different shapes, sizes, and locations, one can simulate the occurrence of disease, compensation, and vascular intervention.

$\mathrm{MB}$ is not suited to the flow pattern induced by a patent $\mathrm{SB}$. This mismatch between bifurcation geometry and global flow pattern creates a flow disturbance that may then lead to an accelerated renarrowing of the MB lumen.

This study attempts to describe these sorts of interactions through an integrated approach. Novel dynamic benchtop fluidmechanical models were used to describe the flow patterns at different points along the time axis including the healthy, diseased, and after-intervention states. A new animal model was then developed to test the predictions based on these benchtop models and to describe the in vivo sequelae stemming from the demonstrated flow disturbances. By percutaneously occluding the SB of animal bifurcations and comparing to bifurcations in which the SB was left patent, the effects of SB occlusion on MB stent intimal hyperplasia were described. Acute in vivo studies to quantify leukocyte involvement were then used to propose a mechanism for the observed trade-off between SB patency and MB long-term result.

\section{Methods}

Flow models. Scaled three-dimensional models of generalized largeartery $(\sim 3 \mathrm{~mm})$ bifurcations were created using sculptors' microcrystalline wax. These models were then recreated in poly(ethylene glycol) (PEG) mol wt 1,500 by a reverse-casting technique with a polyurethane cast. The PEG models were suspended inside a large container that was then filled with a silicone compound (RTV 6166; GE Silicones, Wilton, Connecticut, USA). After allowing the silicone to cure, the entire container was heated, melting out the PEG and leaving a clear silicone block with evacuated flow channels in the shape of the original bifurcation. Using a similar technique, shaped blind-ended cavities were created in the same silicone block adjacent to the flow channels. When these cavities were pressurized, they would impinge on the adjacent flow channel. Conversely, when the cavities were depressurized, they would pull outward on the wall of the adjacent flow channel. By combining several of these cavities of different shapes, sizes, and locations (Figure 1), we could simulate continuous dynamic changes in the arterial geometry including concentric and eccentric lesions, aneurysms, degrees of taper, and outward remodeling.

Parametric comparison. In practice, arterial bifurcations vary in shape and size. The differences in these geometries might influence the applicability of the studied effect. To demonstrate an approach toward this problem, important parameters such as bifurcation angle and relative $\mathrm{MB} / \mathrm{SB}$ ratio were defined. Families of models were then constructed while varying one of these parameters to see in what way the results obtained were influenced by the changes. For example, to investigate the effect of $\mathrm{MB} / \mathrm{SB}$ diameter ratio, three models were built possessing a small-, medium-, and large-SB (2:1, 4:3, 1:1 $\mathrm{MB} / \mathrm{SB}$ diameter ratio, respectively).

Analysis. Models were pulsatile-perfused using a water-silicone mixture to ensure dimensionless similarity of both the Reynolds $(\sim 300)$ and the Womersley $(\sim 4.7)$ parameters. Small (less than $1 / 30$ of vessel diameter) neutral-buoyancy microspheres were fabricated from a mixture of polystyrene and poly(methyl methacrylate). These microspheres were injected proximally into the flow and filmed orthogonally using a digital camera (Sony Corp., Tokyo, Japan). Successive digital frames were superimposed after background subtraction, to create a streakline image of particle paths. Regions of boundary layer separation were identified by streakline departure from the vessel wall, in combination with evidence of reverse flow adjacent to the wall on video.

In vivo models. The porcine animal model was used to establish a relationship between regions of flow disturbance and a higher rate of neointimal hyperplasia. Animal care and procedures were in accordance with the guidelines of the American Association for the Accreditation of Laboratory Animal Care and the NIH. The SB of the right ilio-femoral bifurcation was percutaneously occluded as described later, while the left bifurcation was left intact (Figure $2 \mathrm{~A})$. The animals were then assigned to two groups (Figure $2 \mathrm{~B}$ ). Group A animals were followed for 28 days after SB occlusion, to allow arterial remodeling to occur. After 28 days, the MBs of the bifurcations on both sides were stented using identical stents. Group B animals were followed for only 3 days prior to stenting of the MBs of the bifurcations on both sides using identical stents. This shortened period between SB occlusion and MB stenting was assumed not to allow $\mathrm{MB}$ compensatory remodeling to occur. Both

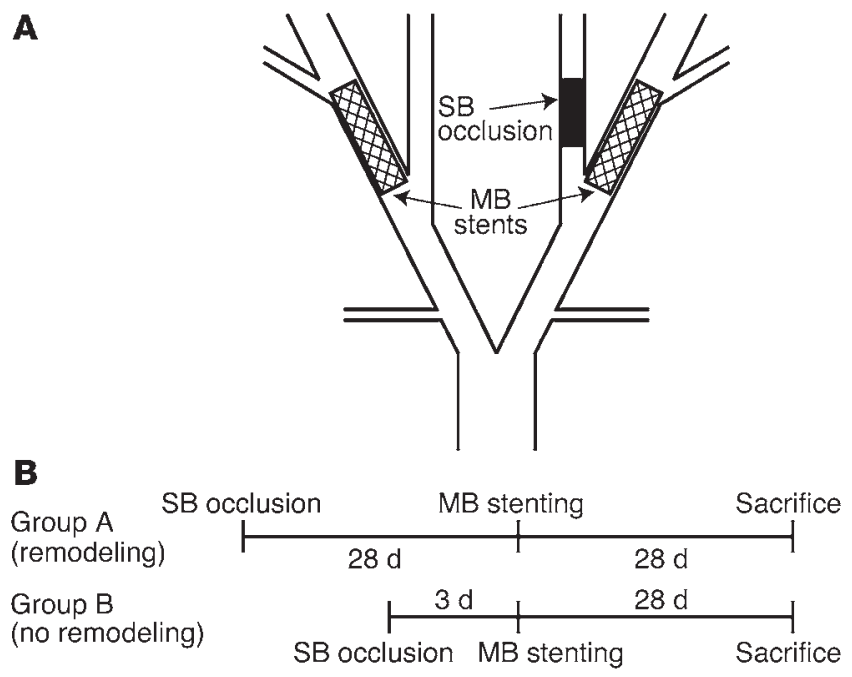

\section{Figure 2}

In vivo model. Unilateral occlusion of the SB of the porcine ilio-femoral bifurcation was performed as described $(\mathbf{A})$. The MBs were then stented bilaterally with or without allowing the MB time to adapt to SB occlusion (B). After chronic follow-up, MB stents were analyzed for neointimal hyperplasia. Each animal served as its own internal control, with comparisons made between neointimal hyperplasia on the SBoccluded (right) side versus the SB-patent (left) side. 


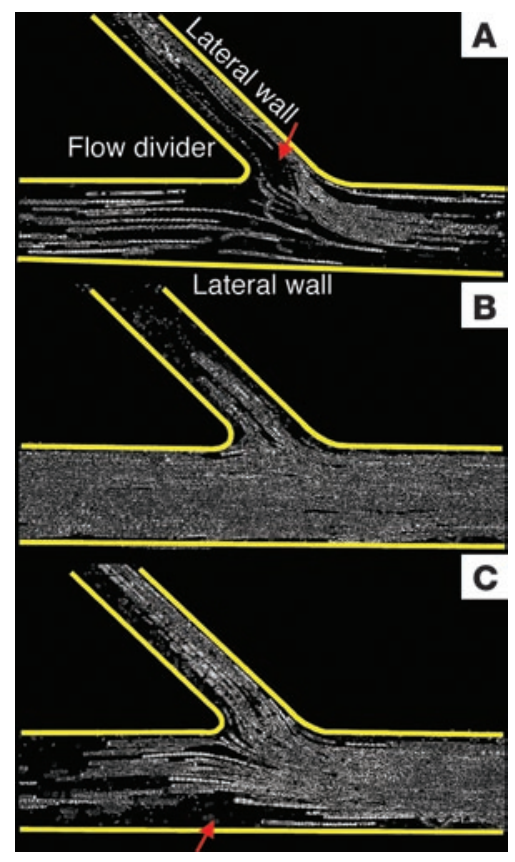

Figure 3

Flow disturbance in vitro. Flow patterns through different bifurcation states were visualized by streaklines at peak flow. (A) In a healthy, nonoccluded bifurcation, there is a large region of boundary layer separation adjacent to the lateral wall of the SB (arrow). However, optimization of MB geometry prevents the occurrence of boundary layer separation in the MB. (B) When the SB is occluded, there is uniform, nondisturbed flow in the reoptimized MB. (C) When the SB is dilated while holding MB geometry fixed, a new large region of boundary layer separation is introduced into the MB on the lateral wall (arrow).

groups were then followed for an additional 28 days, at the end of which the animals were euthanized and the bifurcations excised and processed for histological analysis. Immunohistological and morphometric measurements were performed on the MB stents, with each animal serving as its own internal control by comparison between the SB-occluded and the SB-patent sides.

$S B$ occlusion. Percutaneous SB occlusion was performed by partially deploying a long stent in the SB such that the distal end of the stent was left unexpanded, thus forming a funnel of struts in the center of the lumen. This funnel was then injected with polymer microspheres approximately $1 \mathrm{~mm}$ in diameter and consisting of $70 \%$ polystyrene and $30 \%$ poly(methyl methacrylate), via the guiding catheter. The microspheres became entrapped within the "funnel" portion of the stent. Over the course of the next 1-2 days, the spaces between the spheres thrombosed, thus producing a stable chronic occlusion of the SB.

Histological and morphometric analysis. After sacrifice, the entire bifurcations were dissected free, pressure-perfused, and fixed in $10 \%$ neutral buffered formalin. Following ethanol and xylene processing, the entire bifurcations were embedded whole in a methyl methacrylate/ butyl methacrylate resin (Polysciences Inc., Warrington, Pennsylvania, USA) while taking care to preserve the original bifurcation geometry. A copper rod was embedded alongside the $\mathrm{MB}$ of the bifurcations to serve as a reference point for the lateral wall of the $\mathrm{MB}$ in the subsequent morphological analysis. Following UV polymerization, serial cross-sectional planes along the length of the stents were precision-sawed, then microtome-cut at $5 \mu \mathrm{m}$ thickness and stained with Verhoeff's elastin and H\&E stains. Leukocyte density was quantified by morphology and validated by immunostaining for porcine CD45 (allotypic variant; Serotec Ltd., Oxford, United Kingdom) with low-temperature antigen retrieval and a tyramide signal amplification system (DakoCytomation California Inc., Carpinteria, California, USA). Smooth-muscle cell density was determined using SMC $\alpha$-actin stain. ECM content was defined using Carstairs' fibrin stain. Computer-assisted digital planimetry measured lumen, neointimal, and medial areas and neointimal thickness.

\section{Results}

Models. Blood flow through an arbitrary bifurcating geometry introduced large areas of boundary layer separation in the main and daughter branches (data not shown). However, the flow pattern through a "healthy" bifurcation with a patent SB (Figure 3A) could be optimized to produce little observable flow disturbance in the MB. This was done by subtle manipulation of the MB geometry (primarily the introduction of a slight taper in the lateral wall) and the relative downstream resistances of the two branches. A region of boundary layer separation was still evident in the ostium of the SB. The precise location of this region of separation shifted depending on the SB diameter. However, both the lateral and the flow divider walls of the $\mathrm{MB}$ were devoid of any significant evidence of boundary layer separation or other flow disturbance in the simulated healthy state.

As the SB was gradually constricted to simulate the occurrence of arterial disease, the flow pattern in both branches changed. Initially, total flow through the SB was decreased by $93 \%$. Next, vascular remodeling was simulated by reoptimization of the MB geometry, ultimately leading to the simulated diseased state (Figure 3B). In this state, there was indeed very little perfusion through the now-constricted SB; however, boundary layer separation in the MB did not occur, even in the absence of taper, as can be seen by the filling of virtually the entire cross section by streamlines.

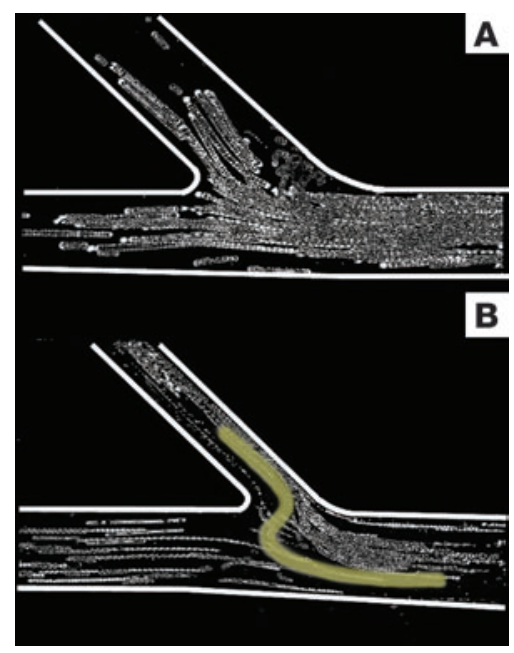

\section{Figure 4}

Parametric analysis. (A) Optimized flow through large-SB model. (B) Optimized flow through small-SB model. Note the location of flow separation in the SB on the lateral wall in $\mathbf{A}$ as opposed to the flow divider in $\mathbf{B}$. Swirling $S$ pattern is highlighted. 

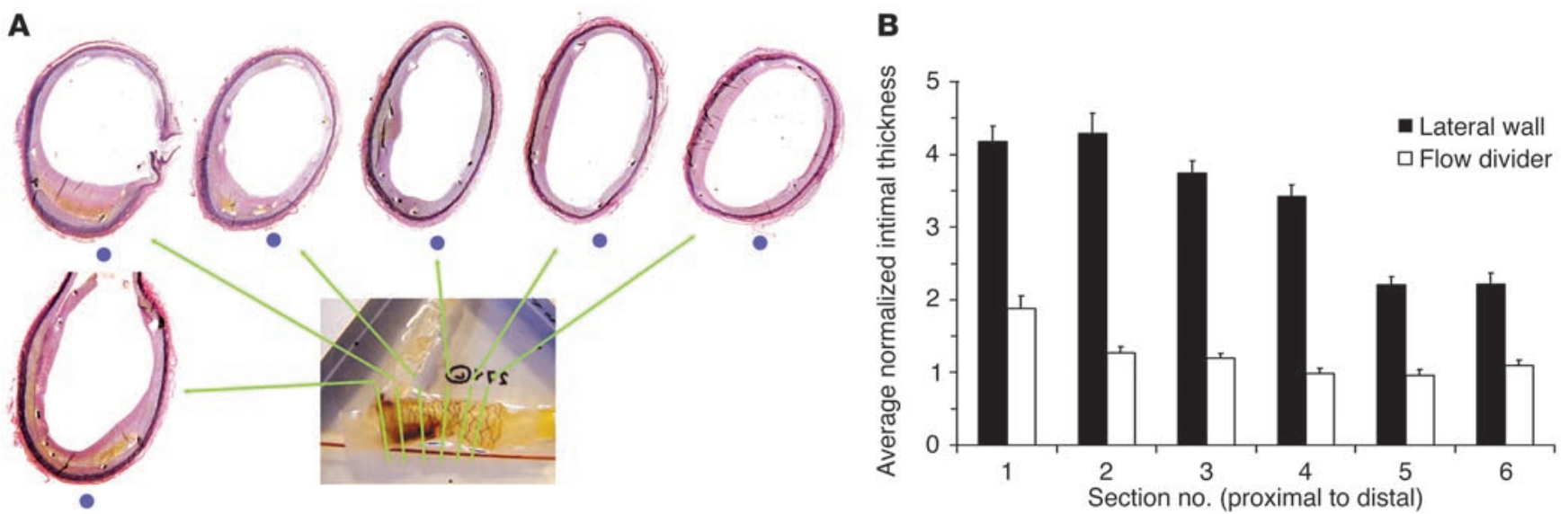

Figure 5

Pattern of neointimal hyperplasia in vivo. (A) Serial sections along a single bifurcation show the pattern of hyperplasia on an MB stent in the SBpatent case. The original bifurcation is shown inset with green lines denoting the longitudinal location of each section. A blue dot next to each section denotes the location of the lateral wall. (B) The average pattern ( $n=11$ animals) recapitulates the individual pattern. Lesions are highly eccentric, with thickness at the lateral wall reaching values 3.5 times higher than those on the flow divider wall. The difference between the two walls grows rapidly in the proximal part of the stent opposite from the SB ostium and then diminishes in the more distal sections.

Finally, clinical intervention was simulated by removing the constriction from the SB without modification of the MB geometry (Figure 3C). When this was done, a mismatch was created between the $\mathrm{MB}$ geometry and the global conditions under which the MB needs to function. The $\mathrm{MB}$, through continuous optimization, had adapted to functioning within the setting of an occluded SB, whereas now it was in the setting of a patent SB. Not surprisingly, this led to a significant region of boundary layer separation in the $\mathrm{MB}$ on the lateral wall opposite from the ostium of the SB and stretching downstream. This region can be expected to be associated with low, oscillatory shear stress, poor mass transfer between the vessel wall and the flowing blood, and increased residence times for cells and other blood-borne particles. The effects of these variables were reflected by the in vivo results.

Geometric parameters. In this initial study, a comparison was made between the effects of dynamic flow alterations in a large- versus a small-SB model (1:1 and 1:2 SB/MB diameter ratio, respectively). Parametric analysis of flow through the bifurcation (Figure 4) demonstrated that flow properties in the MB were for the most part unchanged. In this experiment the $\mathrm{SB} / \mathrm{MB}$ total flow ratio was maintained at $1: 1$ as in the large-SB model by adjusting the downstream resistances accordingly. SB flow pattern, however, exhibited different behavior: Whereas the region of boundary layer separation in the SB was on the lateral wall in the large-SB model, the region clearly shifted to the flow divider in the small-SB model. Particles incident on the flow divider in the small-SB model exhibited a swirling $S$ pattern (Figure 4). This behavior was not observed in the large-SB model.

Chronic in vivo results with remodeling. In vivo investigation showed that the pattern of stent-induced neointimal hyperplasia (Figure 5) closely resembles the area of boundary layer separation seen in the benchtop models (Figure 3C) in extent, shape, and

\section{Figure 6}

location. The injury-induced lesion was highly eccentric, with a maximum at the lateral wall of the MB. In concert with the region of boundary layer separation, the lesions began just distal to the ostium of the SB, grew in extent, and then shrank back to nearly normal as one moved distally along the length of the stent. Quantification was performed by measuring the thickness of the neointimal layer at the lateral and the flow divider wall. All measurements for each animal were normalized by the average thickness of the neointimal layer on the flow divider wall of the middle three sections of the stent (sections 3-5). The average pattern of restenosis (Figure 5B) recapitulated the pattern seen in

\section{A}

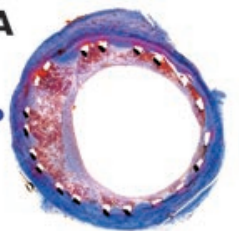

B

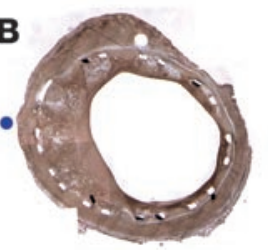

C
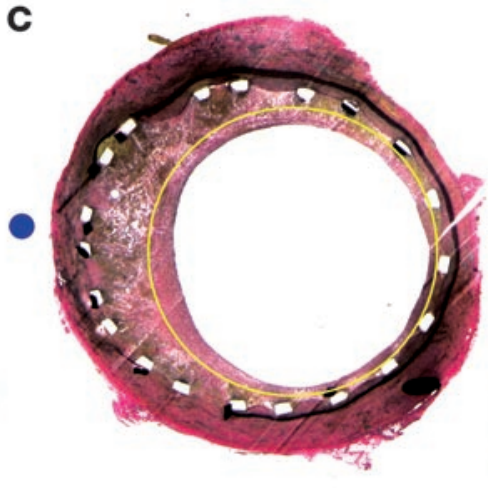

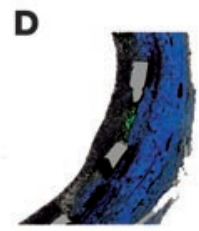

E

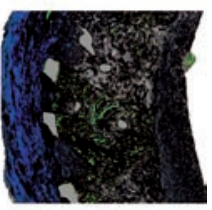

Lesion composition. High-magnification images of one of the sections of a chronic (14-day) lesion. A blue dot in each image denotes the location of the lateral wall. (A) Carstairs' fibrin stain demonstrates high concentrations of fibrin around the stent struts, particularly on the lateral wall. (B) Smooth-muscle cell $\alpha$-actin stain shows tightly packed smooth muscle inside along the lumen (inside of the hoop in $\mathbf{C}$ ), with areas poor in smooth-muscle cells in the crescent region on the lateral wall. (C) Ver Hoeff's elastin stain shows a cellular, dense region of relatively uniform thickness (inside yellow circle) and an ECM-rich, sparse crescent region located on the lateral wall. (D and E) False-colored enhancement of fibrin concentration (green) highlights the difference in ECM away from the struts in the flow divider (D) and the lateral wall (E). 
A

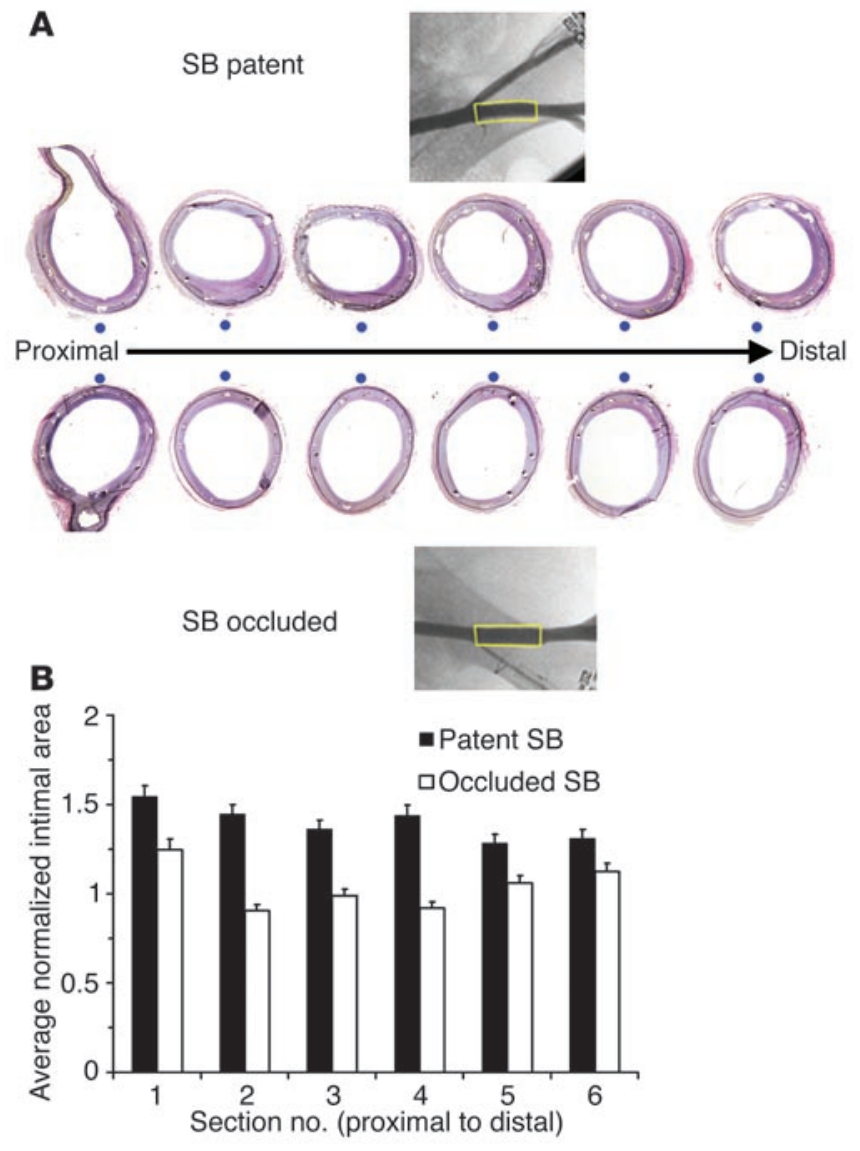

each individual animal. The lesions in the MB were highly eccentric, with neointimal thickness at the lateral wall reaching values of up to 3.5 times higher than those on the flow divider wall. The difference between the two walls grew rapidly to a maximum just distal to the proximal end of the stent (section 2) and then diminished gradually as one moved distally.

Histological evaluation demonstrated that the chronic arterial lesions formed in the stented bifurcations can be divided into two regions, each with distinct shape, cell morphology, and properties (Figure 6). An inner region, roughly annular in shape, is bounded by the lumen on one side and is $200-300 \mu \mathrm{m}$ thick. An outer crescentic region serves as the outer boundary of the annular ring of cells. The inner annular region is distributed evenly around the entire circumference of the lumen, is densely populated with a high concentration of contiguous cells that stain positive for alpha-actin, smooth-muscle cells. This region is poor in ECM and fibrin in particular. The crescentic region, outside of the annular ring of smooth-muscle cells, has its greatest mass on the lateral wall - the side of flow separation. This region was less compact, more highly vascularized, and relatively poor in smooth-muscle cells; moreover, it contained several "holes" almost entire devoid of smooth-muscle cells. In contrast to the annular cell ring, the crescent region is fibrin-rich both around the stent struts and in the regions in between and away from the struts.

Following unilateral SB occlusion, each animal was first analyzed on an individual basis to show the qualitative pattern of neointimal hyperplasia in the SB-occluded versus the SB-patent side (Figure $7 \mathrm{~A})$. The differences in pattern of hyperplasia were immediately

\section{Figure 7}

Effect of SB occlusion on neointimal hyperplasia in group A. (A) Serial sections along the $\mathrm{MB}$ stents of one animal demonstrate the effect of SB occlusion. Blue dots at each section denote the location of the lateral wall. Insets at top and bottom show the original bifurcations in vivo on angiogram with the stent locations outlined in yellow. The stent on the SB-patent side (top) is covered by substantially more eccentrically distributed intimal hyperplasia. (B) The average pattern $(n=3$ animals, $n=6$ bifurcations) reveals that the SB-occluded case incurs substantially less neointimal area than the SB-patent case. The difference between the two cases is most pronounced just distal to the proximal end of the stents and diminishes as one moves distally.

apparent: the volume of neointimal growth on the SB-patent side was significantly larger than on the SB-occluded side. This additional growth was distributed eccentrically centered at the lateral wall. As one moved toward the more distal sections, the two sides became similar. Interanimal variability was normalized by normalizing all intimal thickness to the average thickness of the lesion at the flow divider for sections 2-6 on the right side. For group A animals, the pattern for the occluded-SB case (Figure 7B) was the one normally seen with most stent types. The intimal response was most pronounced at the ends of the stent and minimal in the middle. In contrast, the SB-occluded case exhibited a more uniform pattern. The difference between the two cases grew rapidly in the proximal segment and then shrank toward the distal sections.

Chronic in vivo results without remodeling. When the experiment was repeated while not allowing the MB time to remodel in response to SB occlusion (group B), a completely different picture emerged (Figure 8). In contrast to the clear difference between the SB-occluded and SB-patent cases seen in the presence of remodeling (Figure 7), in the absence of remodeling no such difference could be observed. The SB-occluded and SB-patent cases behaved in a similar manner, both exhibiting the pattern of neointimal hyperplasia previously seen in the SB-patent case (compare Figure 8 with Figure 7B).

Acute in vivo results. A representative section (Figure 9) shows the asymmetric pattern of acute (48 hours after implantation)

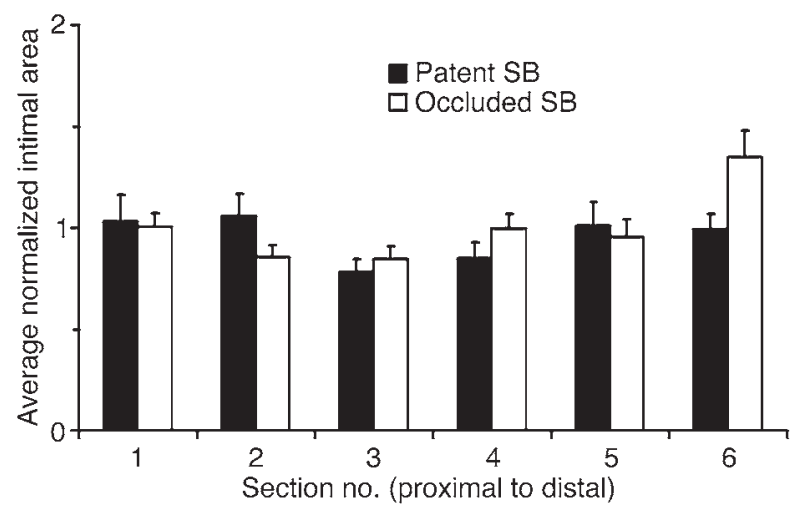

\section{Figure 8}

Occlusion in the absence of remodeling (group B). In group B animals, the MB was not allowed time to adapt to an occluded SB. Thus, a different pattern of intimal hyperplasia occurred. There is no significant difference between MB stent performance in the SBoccluded versus SB-patent case ( $n=3$ animals, $n=6$ bifurcations). The "protective" role that SB occlusion had on the MB stent is lost when remodeling is limited. 


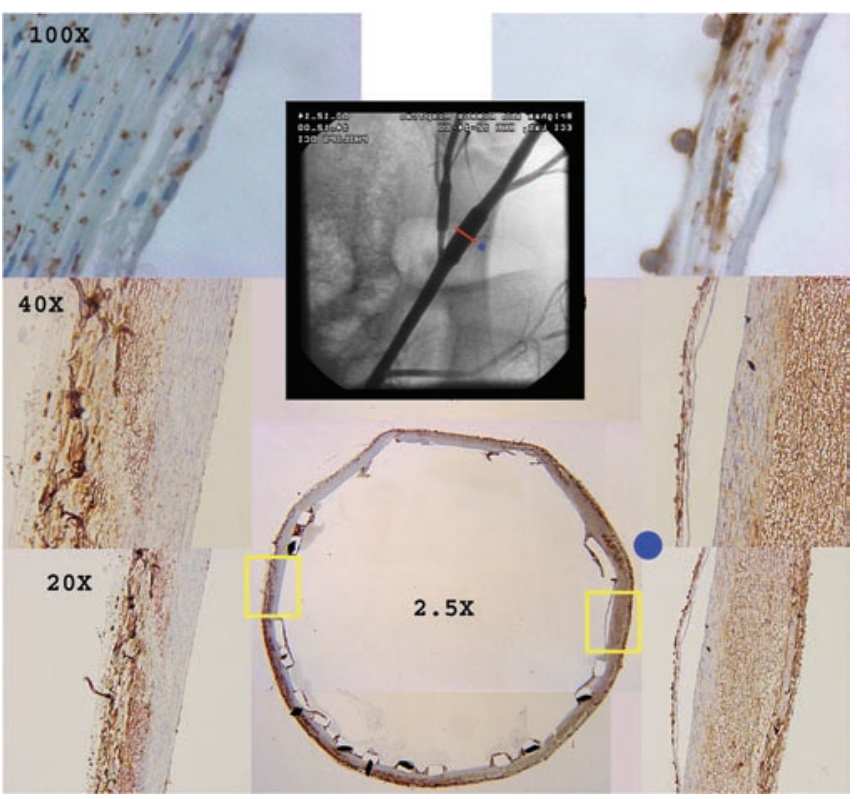

leukocyte adhesion. The lateral wall showed a dense 'carpeting' of macrophages, individually adherent to the vessel wall. As demonstrated by the angular orientation of the section, the region of leukocyte concentration correlates to the region of boundary layer separation noted in the benchtop models. In contrast, the flow divider wall, which exhibited a region of high shear stress in the models, was completely spared of macrophage adhesion at this early stage. The average number of leukocytes (Figure 10A) was dramatically affected by SB occlusion. The number of leukocytes on the SB-patent side increased dramatically toward the middle of the stent, only to shrink back as one moved away from the flow disturbance. In contrast, the number of leukocytes on the SB-occluded side remained fairly constant along the entire length of the stent.

Another important difference in leukocyte accumulation between the SB-occluded and SB-patent sides was the degree of asymmetry between the lateral and the flow divider walls. Leukocyte adhesion on the SB-patent side was highly asymmetric, with the lateral wall exhibiting high numbers of leukocytes and the flow divider wall exhibiting much lower numbers (Figure 10A). Interestingly, the number of leukocytes on the flow divider wall was lower than at both walls on the SB-occluded side. The SB-occluded side, on the other hand, exhibited a symmetric pattern of accumulation,

\section{Figure 10}

Effect of SB occlusion on leukocyte accumulation. (A) Leukocyte count per high-power field on the SB-patent side increases as one moves distally from opposite the ostium of the SB, followed by a decrease toward the distal edge of the stent (in black). In contrast, the SBoccluded side (in dark gray) exhibits a relatively constant rate of leukocyte accumulation along the length of the stent. The abnormal results in the most proximal section are most likely a result of the inaccuracy of stent deployment at this location. (B) The ratio between leukocyte number on the lateral wall and the flow divider wall gives an index of asymmetry. On the patent side (in black), the asymmetry is maximal at the proximal end and decreases distally. On the SB-occluded side (in white), there is very little asymmetry on any section along the length of the stent. The solid line denotes unity.

\section{Figure 9}

Acute monocyte adhesion. CD45 immunostain of artery stented with Palmaz P-108 stent (JJIS, Warren, New Jersey, USA) at 48 hours. The central top panel shows an angiogram of the original bifurcation in vivo. The red line on the angiogram denotes the longitudinal level at which the section was taken. The central bottom panel shows the entire cross section. The blue dot represents the lateral wall. The yellow boxes denote the two regions of interest at the lateral (right) and the flow divider (left) walls. The panels on the right and left show successive magnifications of the regions of interest. Note the carpeting of adherent monocytes on the lateral wall in comparison to the complete absence of monocytes on the flow divider wall at this early time point.

with similar numbers of leukocytes adherent to the lateral and the flow divider walls. Plotting the ratio between the number of leukocytes on the lateral and the flow divider wall for both the occluded and the patent SB cases highlights this difference in asymmetry. This plot (Figure 10B) shows that the maximal degree of asymmetry on the left (SB-patent) side occurred immediately distal to the proximal end of the stent, and then gradually decreased as one moved distally. On the SB-occluded side, the ratio between the accumulations on the lateral and the flow divider walls was close to unity throughout the length of the stent.

\section{Discussion}

Blood flow through a bifurcation introduces disturbances in different areas of the bifurcation geometry. The most prominent of these are regions of boundary layer separation. These show up as areas where the streamlines that begin close to the wall in the proximal segment arc away from the wall at a position known as the "stagnation point." Farther downstream, the streamlines return toward the vessel wall and run parallel to it again from the "point of reattachment." Between the stagnation and the reattachment points, flow

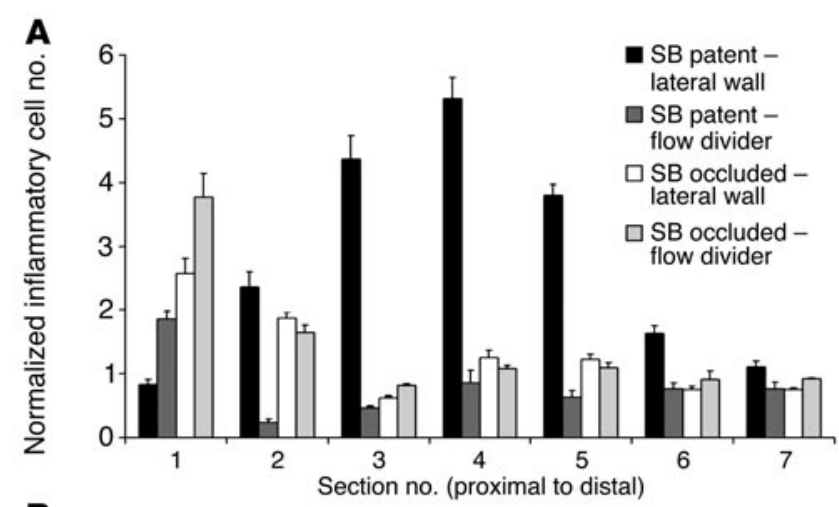

\section{B}

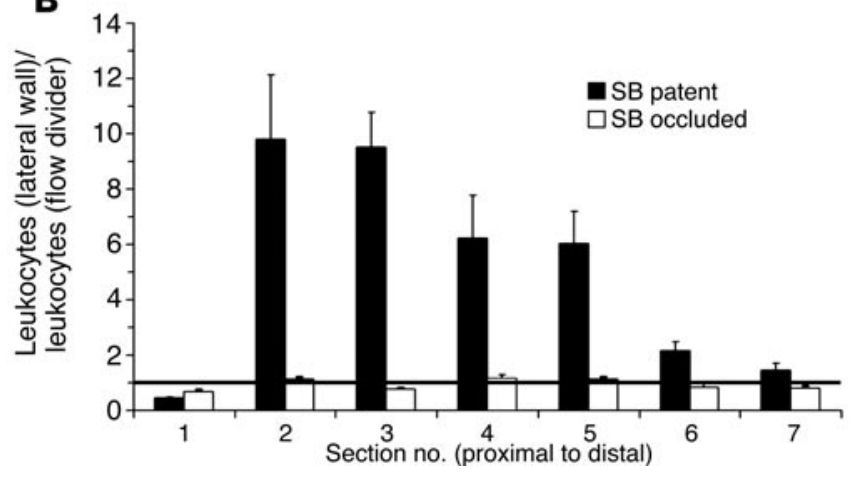


along the wall is in the retrograde direction, thus creating a circular eddy pattern. These regions of boundary layer separation and the mechanical conditions that accompany them have been shown in the past to promote the development of atherosclerotic disease.

In the benchtop models, regions of flow separation, regions of low and high shear, accelerations, and decelerations were all consistent with predictions from basic principles. The location and extent of these flow phenomena changed from one case of the experiment to the other, simulating the flow conditions likely to occur in the course of progression of atherosclerotic disease, compensation by the arterial system, or the effect of mechanical intervention. Flow separation in the MB did not seem to be greatly altered in the parametric comparison between a large- and a small-SB diameter. This suggests that the effects on the MB are not governed by SB diameter but rather by total SB flow, which in our model could be controlled as an independent parameter from SB diameter. Separation in the SB was, however, shown to be qualitatively different in the small-SB case compared with the large-SB case.

The pattern of flow in the MB was directly affected by alterations in the SB alone. At high levels of SB occlusion, nonseparated flow was achieved in the MB even without the introduction of taper. Since the removal of taper in the absence of flow separation physically increases the vessel diameter, thus lowering resistance to flow, adaptive changes in the arterial bed would develop in this direction. Compensatory dilation of the distal portion of the MB should be observed in response to occlusion of the SB (32). Thus a complete (or nearly complete) SB occlusion, would remove the trigger for flow separation in the MB and would essentially convert the $\mathrm{MB}$ into a simple, straight, nonbifurcating vessel. This observation remains to be shown in vivo. Dilation of the SB, on the other hand, re-established a significant region of separation in the MB. The clinical implications of this new region of flow disturbance cannot be demonstrated in the benchtop model but can be predicted on the basis of work in the field relating flow conditions to biological response, and was correlated to the in vivo results obtained here. We note, however, that human vascular disease and response to injury is affected by many variables other than flow conditions. It is not our intention here to make definitive recommendations about the course of action in a clinical setting but rather to present the role that dynamic flow interactions play in this setting and their ability to at least partially address some of the common observations seen in this field.

The morphology of the chronic restenotic lesion correlates strikingly with the location, shape, and extent of flow disturbance seen in the benchtop models. The lesions in the patent-SB case all begin immediately opposite the ostium of the SB, are all located eccentrically on the lateral wall, and all diminish as one proceeds distally along the stent length. The chronic hyperplastic response observed here can be divided into two distinct regions: a symmetric, uniform, cellular, ECM-poor "annular region" and an eccentric, nonuniform, sparsely cellular, ECM-rich "crescentic region." The differences in morphology between these two regions, particularly in light of the differences in flow conditions demonstrated by the benchtop models, suggests two distinct cellular processes at work. One explanation for these observations that is particularly appealing would be to ascribe the cellular processes in the crescentic region to the effects of flow disturbance, whereas the processes in the annular region would reflect a vascular cellular response to mechanical trauma, irrespective of flow. Other explanations exist, and full elaboration of these events are the topic of further study.
The good correlation between generalized physical models and in vivo results is an important finding in and of itself. Making a priori predictions using generalized models is challenging, since local geometric variation and secondary flow phenomena are important in determining the detailed flow conditions. However, the successful prediction based on generalized flow models presented here implies that the primary flow conditions such as boundary layer separation determine the overall pattern of tissue response. Furthermore, the link between flow patterns in general and boundary layer separation in particular and intimal hyperplasia has been well established for the primary disease of atherosclerosis. While restenosis shares many common aspects with atherosclerosis, they are not one and the same. Restenosis includes the added aspects of a permanent implant and a direct mechanical injury to the vessel wall. The observation that, even in the presence of these aspects, the shape of the restenotic lesion could be predicted on the basis of flow pattern alone, demonstrates the importance of flow phenomena relative to other confounding factors. Viewed differently, the success of generalized models in making certain accurate in vivo predictions implies that modeling work can in fact be applied to a wide variety of real-world patient geometries without the need to individually model each individual case.

Occlusion of the SB in the bifurcation had a profound effect on the extent and geometric pattern of hyperplasia in the MB. SB occlusion essentially eliminated the adverse effect that the bifurcation has on the extent of hyperplasia, reducing it instead to what one would expect to see in a normal nonbifurcating vessel. This finding is not surprising given that SB occlusion essentially converts $\mathrm{MB}$ flow into that which would be seen in a normal nonbifurcating vessel. However, it does underscore that the unique flow conditions in a bifurcation are what predispose it to a suboptimal result rather than the mechanical intervention itself or local differences in biology at the bifurcation. Thus, SB occlusion serves in a protective role with respect to $\mathrm{MB}$ restenosis. Conversely, and perhaps more clinically relevant, the dilation of a SB carried a price in terms of the flow pattern in the MB. In vivo, neointimal hyperplasia in the SB-patent case was greater than the SB-occluded case. The difference between the two cases behaves in the same manner as the difference in flow disturbance, thus providing a mechanistic basis for these observations. One could argue that this difference is not a result of a modulation of the flow pattern per se but rather from changes in the total rate of flow. However, the difference between the SB-patent and the SB-occluded case is axially variable and tends to approach zero at the distal end. This strongly suggests that the difference between the two cases is indeed from a localized proximal flow disturbance in the SB-patent case, as opposed to a modulation of the total rate of flow.

Remodeling played an important role in the effects observed. The same experiment performed with or without allowing the time for remodeling to occur produced vastly different results, in good agreement with the hypothetical and benchtop model predictions. Thus, chronic MB remodeling seems to play a critical role in the "protective" nature of SB occlusion. The precise nature of this remodeling has not been demonstrated here. Whether these are geometric changes, functional cellular changes, other influences, or some combination of all of these cannot be shown on the basis of these data. However, this is a powerful demonstration of the concept that once chronic SB occlusion is present, one can never "return the system to its original state" by a simple mechanical intervention such as angioplasty. 
Leukocytes play a central role in the process of stent-induced neointimal hyperplasia $(33,34)$. Yet the heterogeneous spatial distribution of leukocyte adhesion/transmigration on the level of a single cross section has previously not been demonstrated. The spatial pattern of leukocyte adhesion along the length of the stented segment soon after vascular intervention reflects the cumulative effects of the rate of passive leukocyte arrival, the state of binding-molecule expression, and the forces that act on the leukocyte and vessel wall during the binding process. All of these can be related to the global flow pattern in general and to regions of boundary layer separation in particular. The flow models demonstrate vastly divergent conditions occurring at the lateral wall and the flow divider of the MB of a (patent-SB) bifurcation and thus provide an excellent mechanistic basis for the observed differences in vivo. In the SB-patent case, the region of boundary layer separation on the lateral wall and in particular the low shear stress that can be expected to occur there would provide ideal conditions for leukocytes to adhere. This was borne out by the animal models, which clearly showed a larger number of leukocytes at this location. Conversely, the flow divider wall in the SB-patent case was shown by the flow models to be a region of higher than average shear stress, thus retarding the adhesion of leukocytes. Here, too, the animal models showed a lower than average number of leukocytes. In sum, it seems likely that differential leukocyte adhesion and transmigration is the mechanism that bridges between the early flow disturbance and the late neointimal hyperplasia.
The common clinical practice of SB dilation carries a price - the introduction of a flow disturbance into the MB of the bifurcation, reducing long-term MB patency. Thus, there exists a trade-off between the clinical desire to maximize flow in the short term by dilating the SB and the desire to ensure the patency of the usually more important $\mathrm{MB}$ in the long term. Where feasible, leaving the SB occluded becomes a reasonable strategy in the interest of a more favorable chronic result. Where SB dilation cannot be avoided in the interest of symptomatic relief, introduction of a slight angle of taper into the lateral wall of the MB, either by modification of technique or by design of specialized bifurcation stents, will minimize the resultant disturbance and optimize the long-term result.

\section{Acknowledgments}

This work was supported in part by grants from the NIH (GM/HL 49039, HL 60407, and HL 67246) and an Established Investigator award to E.R. Edelman from the American Heart Association.

Received for publication January 7, 2004, and accepted in revised form February 17, 2004.

Address correspondence to: Yoram Richter, PO Box 58187, Tel Aviv 61581, Israel. Phone: 972-3-767-9000; Fax: 972-3-647-5351; E-mail: yrichter@biorest.co.il.
1. Friedman, M.H., Bargeron, C.B., Deters, O.J., Hutchins, G.M., and Mark, F.F. 1987. Correlation between wall shear and intimal thickness at a coronary artery branch. Atherosclerosis. 68:27-33.

2. Roach, M.R. 1977. The effects of bifurcations and stenoses on arterial disease. In Cardiovascular flow dynamics and measurements. N.H. Hwang and N.A. Normann, editors. Baltimore, University Park Press. 489-539.

3. Malcolm, A.D. 1975. Flow phenomena at bifurcations and branches in relation to human atherogenesis: a study in a family of glass models. Master's thesis. University of Western Ontario. London, Ontario, Canada. 204 pp.

4. Fox, J.A., and Hugh, A.E. 1966. Localization of atheroma: a theory based on boundary layer separation. Br. Heart J. 28:388-399.

5. Lefevre, T., et al. 2000. Stenting of bifurcation lesions: classification, treatments, and results [see comments]. Catheter Cardiovasc. Interv. 49:274-283.

6. Carrie, D., et al. 1998. Coronary stenting of bifurcation lesions using "T" or "reverse Y" configuration with Wiktor stent. Am. J. Cardiol. 82:1418-1421, A1418.

7. Baim, D. 1996. Is bifurcation stenting the answer? Cathet. Cardiovasc. Diagn. 37:314-316.

8. Chevalier, B., Glatt, B., Royer, T., and Guyon, P. 1998. Placement of coronary stents in bifurcation lesions by the "culotte" technique. Am. J. Cardiol. 82:943-949.

9. Cohen, D.J., and Baim, D.S. 1999. Considerations in managing side branches "jailed" by coronary stenting: insights from in vitro studies [editorial; comment]. Catheter Cardiovasc. Interv. 48:235-236.

10. Kobayashi, Y., et al. 1998. Modified "T" stenting: a technique for kissing stents in bifurcational coronary lesion. Cathet. Cardiovasc. Diagn. 43:323-326.

11. Schampaert, E., Fort, S., Adelman, A.G., and Schwartz, L. 1996. The V-stent: a novel technique for coronary bifurcation stenting. Cathet. Cardiovasc. Diagn. 39:320-326.
12. Pan, M., et al. 2002. A stepwise strategy for the stent treatment of bifurcated coronary lesions. Catheter Cardiovasc. Interv. 55:50-57.

13. Al Suwaidi, J., et al. 2001. Immediate and one-year outcome in patients with coronary bifurcation lesions in the modern era (NHLBI dynamic registry). Am. J. Cardiol. 87:1139-1144.

14. Sheiban, I., et al. 2000. Immediate and long-term results of " $\mathrm{T}$ " stenting for bifurcation coronary lesions. Am. J. Cardiol. 85:1141-1144, A1149.

15. Yamashita, T., et al. 2000. Bifurcation lesions: two stents versus one stent - immediate and follow-up results. J. Am. Coll. Cardiol. 35:1145-1151.

16. Carlier, S.G., et al. 1999. Stenting with a true bifurcated stent: acute and mid-term follow-up results. Catheter Cardiovasc. Interv. 47:361-396.

17. Cervinka, P., et al. 2000. Coronary bifurcation stenting using dedicated bifurcation stents. Catheter Cardiovasc. Interv. 49:105-111.

18. Bharadvaj, B.K., Mabon, R.F., and Giddens, D.P. 1982. Steady flow in a model of the human carotid bifurcation. Part I - flow visualization. J. Biomech. 15:349-362.

19. Bharadvaj, B.K., Mabon, R.F., and Giddens, D.P 1982. Steady flow in a model of the human carotid bifurcation. Part II - laser-Doppler anemometer measurements. J. Biomech. 15:363-378.

20. Asakura, T., and Karino, T. 1990. Flow patterns and spatial distribution of atherosclerotic lesions in human coronary arteries. Circ. Res. 66:1045-1066

21. Pritchard, W.F., et al. 1995. Effects of wall shear stress and fluid recirculation on the localization of circulating monocytes in a three-dimensional flow model. J. Biomech. 28:1459-1469.

22. Sabbah, H.N., Khaja, F., Brymer, J.F., Hawkins, E.T., and Stein, P.D. 1984. Blood velocity in the right coronary artery: relation to the distribution of atherosclerotic lesions. Am. J. Cardiol. 53:1008-1012.

23. Texon, M. 1957. A hemodynamic concept of atherosclerosis, with particular reference to coronary occlusion. A.M.A. Arch. Intern. Med 99:418-427.

24. He, X., Ku, D.N., and Moore, J.E., Jr. 1993. Simple calculation of the velocity profiles for pulsatile flow in a blood vessel using Mathematica [erratum 1993, 21:557-558]. Ann. Biomed. Eng. 21:45-49.

25. Moore, J.E., Jr., Xu, C., Glagov, S., Zarins, C.K., and $\mathrm{Ku}$, D.N. 1994. Fluid wall shear stress measurements in a model of the human abdominal aorta: oscillatory behavior and relationship to atherosclerosis. Atherosclerosis. 110:225-240.

26. Kuban, B.D., and Friedman, M.H. 1995. The effect of pulsatile frequency on wall shear in a compliant cast of a human aortic bifurcation. J. Biomech. Eng. 117:219-223.

27. He, X., and Ku, D.N. 1996. Pulsatile flow in the human left coronary artery bifurcation: average conditions. J. Biomech. Eng. 118:74-82.

28. Fernandez, R.C., De Witt, K.J., and Botwin, M.R. 1976. Pulsatile flow through a bifurcation with applications to arterial disease. J. Biomech. 9:575-580.

29. Friedman, M.H., Kuban, B.D., Schmalbrock, P., Smith, K., and Altan, T. 1995. Fabrication of vascular replicas from magnetic resonance images. J. Biomech. Eng. 117:364-366.

30. Rindt, C.C., and Steenhoven, A.A. 1996. Unsteady flow in a rigid 3-D model of the carotid artery bifurcation. J. Biomech. Eng. 118:90-96.

31. Yoganathan, A.P., et al. 1986. Steady flow velocity measurements in a pulmonary artery model with varying degrees of pulmonic stenosis. J. Biomech. 19:129-146.

32. Langille, B.L. 1996. Arterial remodeling: relation to hemodynamics. Can. J. Physiol. Pharmacol. 74:834-841.

33. Ross, R. 1999. Atherosclerosis is an inflammatory disease. Am. Heart J. 138:S419-S420.

34. Danenberg, H.D., et al. 2002. Macrophage depletion by clodronate-containing liposomes reduces neointimal formation after balloon injury in rats and rabbits. Circulation. 106:599-605. 